\title{
Vólvulo agudo de la vesícula biliar: tratamiento laparoscópico en un lactante
}

\author{
Acute gallbladder volvulus: laparoscopic treatment in an infant \\ Carlos García-Hernández ${ }^{1,2 *}$, Christian Archivaldo-García ${ }^{1,2}$, Lourdes Carvajal-Figueroa ${ }^{1,2}$, \\ Madelinne Granados-Macías ${ }^{3,4}$ e Israel Herrera-Flores ${ }^{3,4}$ \\ ${ }^{1}$ Departamento de Cirugía Pediátrica, Hospital Infantil Privado; ${ }^{2}$ Alta Especialidad en Cirugía de Mínima Invasión en Pediatría, Universidad Autónoma \\ de México; ${ }^{3}$ Departamento de Radiología e Imagen, Hospital Infantil Privado; ${ }^{4}$ Alta Especialidad en Radiología Pediátrica, Posgrado, Universidad \\ Autónoma de México. Ciudad de México, México
}

\begin{abstract}
Resumen
Introducción: El vólvulo de la vesícula biliar es una entidad rara en pediatría, de diagnóstico difícil, y el retraso de su tratamiento produce complicaciones. Se presenta el caso de un paciente masculino de nueve meses resuelto por laparoscopia. Caso clínico: 12 horas de evolución con irritabilidad, vómito, fiebre; abdomen doloroso; leucocitosis; y ultrasonido líquido perivesicular. La tomografía computarizada reveló acodamiento del cístico, aumento de la densidad y reforzamiento con el medio de contraste. La laparoscopia encontró vesícula volvulada y necrosada. Discusión: Es una enfermedad poco frecuente. Éste es un niño de nueve meses. El abordaje por mínima invasión permitió establecer el diagnóstico y tratamiento con seguridad.
\end{abstract}

PALABRAS CLAVE: Vólvulo. Vesícula biliar. Laparoscopia.

\begin{abstract}
Introduction: Gallbladder volvulus is a rare and difficult to diagnose pediatric entity, its delayed treatment leads to complications. A 9 months old male case solved by laparoscopy is reported. Case report: Twelve hours of development with irritability, vomiting and fever. Painful abdomen, leukocytosis and ultrasound with perivesicular fluid. Computed tomography revealed cystic bending, increased density and reinforcement of contrast medium. Gallbladder was found volvulated and necrotized by laparoscopy. Discussion: It is a very rare disease. A 9 months old baby was reported in whom a minimally invasive approach allowed diagnosis and safe treatment.
\end{abstract}

KEY WORDS: Volvulus. Gallbladder. Laparoscopy.

\section{Introducción}

El dolor abdominal es un síntoma común en los niños y puede deberse a múltiples causas quirúrgicas y no quirúrgicas. Su origen varía con la edad. En los lactantes, las principales causas de abdomen agudo son: invaginación intestinal, mala rotación intestinal, apendicitis y enfermedad de Hirschsprung".

La afección biliar es poco frecuente en la edad pediátrica y cuando se presenta se relaciona con ciertas alteraciones como la enfermedad de Kawasaki,

\author{
Correspondencia: \\ *Carlos García-Hernández \\ Circuito Valle Dorado, 12 \\ Col. Loma de Valle Escondido \\ C.P. 52930, Atizapán de Zaragoza, Edo. de México, México \\ E-mail: carloscirped@ hotmail.com
}

Fecha de recepción: 02-10-2018

Fecha de aceptación: 14-02-2019

DOI: $10.24875 / \mathrm{CIRU} .19000787$
Cir Cir. 2019;87(S1):58-61

Contents available at PubMed www.cirugiaycirujanos.com 
infecciones sistémicas graves, resecciones ileales o uso prolongado de nutrición parenteral ${ }^{2,3}$.

El vólvulo de la vesícula biliar se define como la torsión del órgano sobre su mesenterio a lo largo del eje del conducto y arteria cística, lo que se ha informado que ocurre con mayor frecuencia en mujeres ancianas ${ }^{4,5}$.

Desde el primer informe sobre la torsión de la vesícula biliar en un adulto por Wendel en 1898 se han descrito alrededor de 400 casos de esta enfermedad'. Se han observado algunos casos en adultos jovenes, pero es muy raro en niños ${ }^{4,6}$. Daux describió en 1925 el primer caso en la edad pediátrica y desde entonces se han notificado 25 casos en las publicaciones médicas inglesas y 38 casos en Japón; el paciente más joven tenía dos años ${ }^{6,7}$.

La baja incidencia de la enfermedad hace que su diagnóstico sea dificil de establecer de manera preoperatoria, pero el retraso terapéutico puede dar lugar a complicaciones graves peritoneales ${ }^{4}$.

El objetivo de este trabajo es notificar el caso de un paciente masculino de nueve meses de edad con un abdomen agudo secundario a un vólvulo de la vesícula biliar, describir los hallazgos radiológicos que permiten establecer un diagnóstico preoperatorio adecuado, así como su resolución satisfactoria mediante cirugía de mínima invasión.

\section{Caso clínico}

Se trata de un paciente masculino de nueve meses de edad, producto de la primera gestación y de un embarazo de evolución normal, obtenido por cesárea a término con peso de $2250 \mathrm{~g}$ y Apgar de 8-9, con crecimiento y desarrollo adecuados para la edad.

El padecimiento actual inició 12 horas antes de su ingreso con cuadro de irritabilidad, vómito de contenido gastroalimentario 6 en 24 horas, sin evacuaciones, fiebre cuantificada en $38^{\circ} \mathrm{C}$ y taquicardia. A la exploración física se halla irritable, con regular estado de hidratación, febril, taquicárdico, abdomen doloroso a la palpación media y profunda con signo de rebote dudoso y peristalsis disminuida.

Se obtuvieron biometría hemática, química sanguínea con pruebas de función hepática, tiempos de coagulación y examen general de orina. El único resultado anormal fue la cifra de leucocitos de 20400 leucocitos/ml (normal, 5000 a 10000 leucocitos $/ \mathrm{ml}$ ); la cuenta diferencial con neutrófilos fue de $65 \%$.

Se realizó una radiografía simple de abdomen que se interpretó sólo con abundante material de residuo. El ultrasonido de hígado y vías biliares identificó

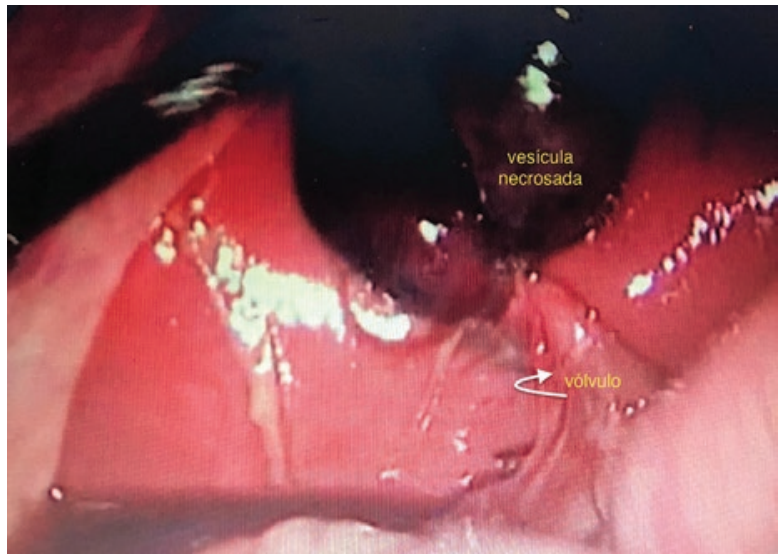

Figura 1. Vesícula biliar necrosada con vólvulo de $720^{\circ}$ en el conducto y arteria cística.

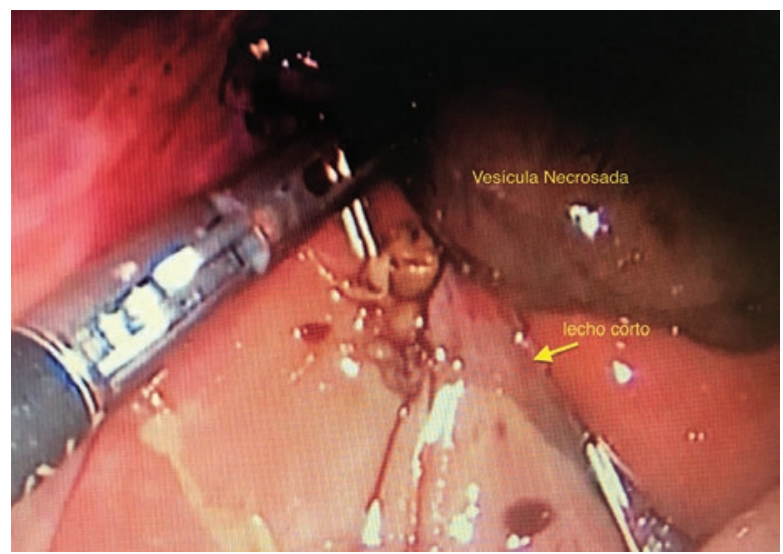

Figura 2. Lecho corto que favoreció el vólvulo de la vesícula biliar.

vesícula biliar distendida, dimensiones de $43 \times 19 \times$ $21 \mathrm{~mm}$, con acodamiento de la vesícula biliar respecto del conducto cístico, y aumento del grosor de la pared anterior hacia el cuello que mide hasta $6.8 \mathrm{~mm}$ y líquido perivesicular.

La tomografía simple y contrastada de abdomen mostró la vesícula biliar distendida, con engrosamiento difuso de su pared de $6 \mathrm{~mm}$, líquido libre perivesicular acumulado de predominio en fosa cística, acodamiento del cístico, aumento de la densidad y reforzamiento tras la aplicación de medio de contraste.

Por persistencia de los síntomas del paciente y con diagnóstico de abdomen agudo de causa indeterminada se practicó una laparoscopia diagnóstica. Con técnica abierta se colocó trócar de $5 \mathrm{~mm}$ umbilical con técnica abierta, con presión de $8 \mathrm{mmHg}$ y flujo de $1 \mathrm{~L} / \mathrm{min}$, y se encontró líquido libre en la corredera parietocólica derecha, el apéndice cecal y el resto del intestino de apariencia normal. Al continuar la revisión se encontró vesícula biliar necrosada con líquido perivesicular purulento y torsión de la 
vesícula biliar de $720^{\circ}$, en sentido dextrógiro sobre su mesenterio y en el eje del conducto y la arteria cística (Fig. 1). Después de corregido el vólvulo se disecó el conducto cístico y se colocó doble grapa de titanio, en una porción sana de éste, se disecó la arteria cística, se colocó doble grapa de titanio y se cortó. Se separó la vesícula de su lecho, el cual era muy corto (Fig. 2). Se extrajo la vesícula en una bolsa. El tiempo quirúrgico fue de 30 minutos, sin sangrado ni complicaciones; se inició la vía oral a las seis horas del postoperatorio y se egresó al quinto día con tratamiento antibiótico. El seguimiento a seis meses no ha mostrado complicaciones.

Los hallazgos histopatológicos fueron vesícula biliar con edema de pared y congestión mucosa con colecistitis gangrenosa aguda y necrosis extensa de la pared.

\section{Discusión}

El vólvulo de la vesícula biliar es una enfermedad poco frecuente que se presenta en mujeres mayores de 70 años. Existen pocas publicaciones de esta enfermedad en pacientes pediátricos; hasta ahora, un niño de dos años de edad ha sido el paciente más pequeño descrito ${ }^{6,7}$.

En este caso se trató de un niño de nueve meses de edad, lo que demuestra que esta anomalía se puede presentar en cualquier momento de la vida. Se desconoce su origen; en los adultos se ha mencionado a la atrofia grasa con aumento de la elasticidad tisular ${ }^{4}$. En los niños se ha relacionado con la ausencia de los ligamentos coronario o triangular ${ }^{7}$, pero en este caso se identificó una fijación muy pequeña de la vesícula al hígado como la causa que favoreció el vólvulo del órgano.

El abdomen agudo en los lactantes rara vez tiene su origen en la afección biliar, por lo que establecer el diagnóstico de manera preoperatoria es muy difícil. El principal síntoma de este paciente fue irritabilidad, en relación con signos dudosos de abdomen agudo en la exploración física y presencia de leucocitosis, que no permitían incluso determinar con certeza el diagnóstico.

Estos síntomas tan poco específicos pueden propiciar un retraso en el tratamiento y con ello complicaciones graves, como la perforación de la vesícula necrosada con desarrollo de peritonitis biliar.

Con el hallazgo de vólvulo de la vesícula biliar se analizaron los estudios radiológicos y se hallaron los siguientes datos que pudieron establecer el diagnóstico de manera preoperatoria: la radiografía simple de

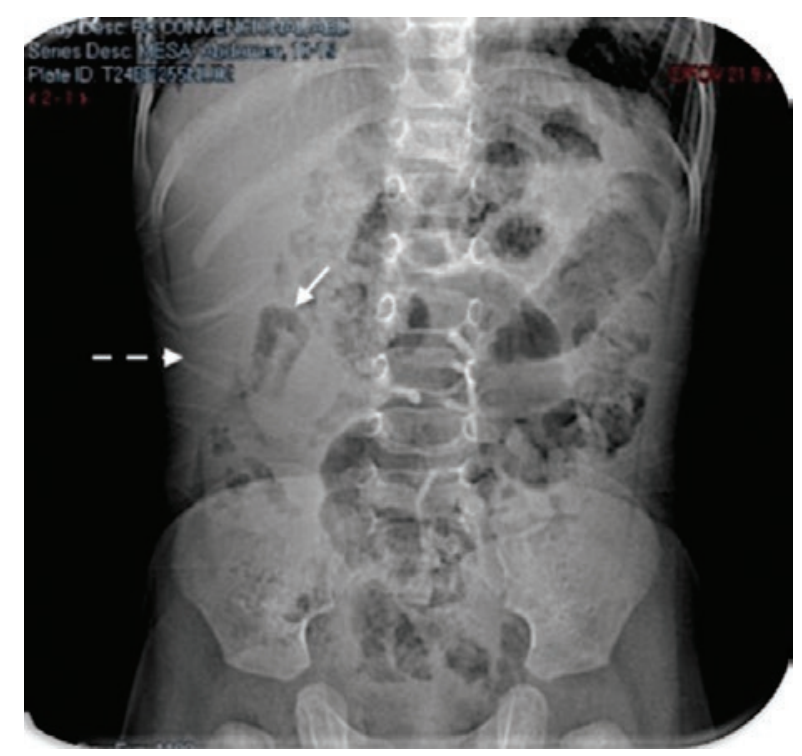

Figura 3. Radiografía simple de abdomen que muestra borramiento de la grasa preperitoneal con compresión del colon transverso.

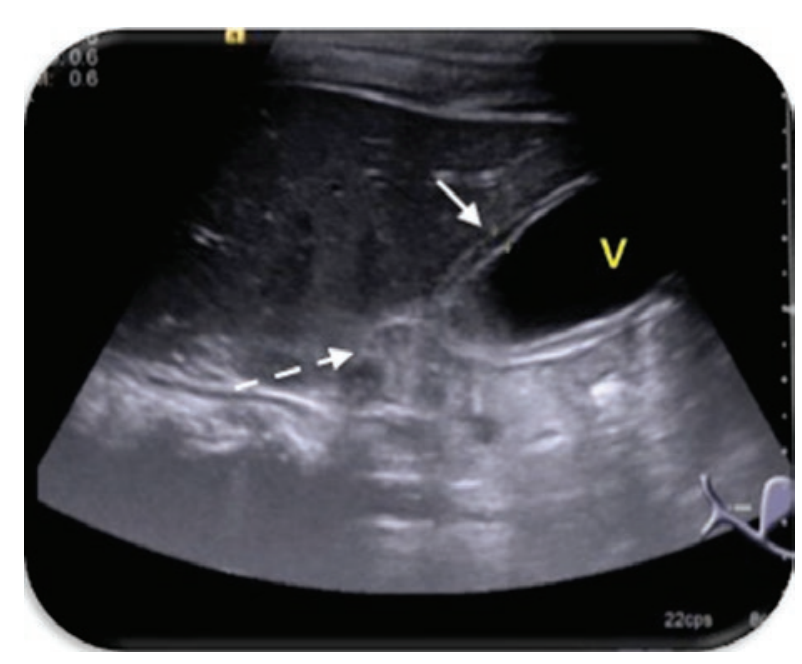

Figura 4. Ultrasonido de hígado y vías biliares (flecha, vesícula). Vesícula fuera de su fosa anatómica normal, con una estructura cónica ecogénica que corresponde al pedículo torcido.

abdomen mostró aumento de la densidad hacia la topografía del polo inferior renal derecho y borramiento de la grasa preperitoneal ipsolateral adyacente al borde inferior del lóbulo hepático derecho, con compresión del colon transverso, sin aire libre (Fig. 3).

El ultrasonido delineó acodamiento de la vesícula biliar con respecto al conducto cístico, con aumento del grosor de la pared anterior hacia el cuello y líquido perivesicular. Esto indicaba que la vesícula biliar no estaba unida al hígado. Además, la vesícula biliar se hallaba fuera de su fosa anatómica normal, con una zona hipoecogénica continua entre las dos capas ecogénicas de la pared y una estructura cónica ecogénica que corresponde al pedículo torcido (Fig. 4). 


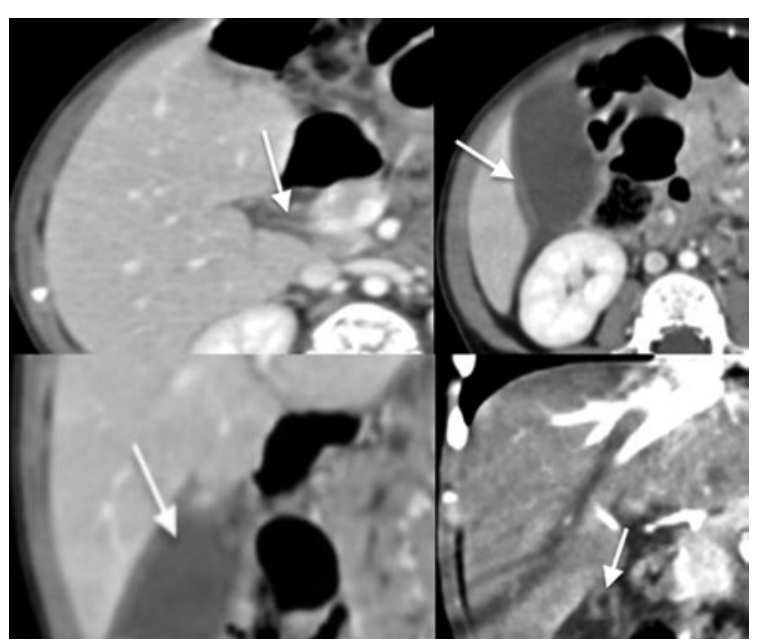

Figura 5. Tomografía de abdomen que muestra líquido libre perivesicular acumulado de predominio en fosa cística, acodamiento del cístico, aumento de la densidad y reforzamiento tras la aplicación del medio de contraste.

Y, por último, la tomografía de abdomen identificó distensión de la vesícula biliar, con engrosamiento difuso de su pared, líquido libre perivesicular acumulado de predominio en la fosa cística, acodamiento del cístico, aumento de la densidad y reforzamiento tras la aplicación de medio de contraste (Fig. 5).

Al considerar los datos mencionados era posible determinar el diagnóstico preoperatorio de vólvulo de la vesícula biliar. El abordaje de mínima invasión permitió establecer el diagnóstico con certeza, así como poder resecar la vesícula necrosada de una manera segura que favoreció la adecuada recuperación de este paciente.

\section{Conflicto de intereses}

Los autores declaran que no existe conflicto de intereses.

\section{Responsabilidades éticas}

Protección de personas y animales. Los autores declaran que para esta investigación no se han realizado experimentos en seres humanos ni en animales.

Confidencialidad de los datos. Los autores declaran que han seguido los protocolos de su centro de trabajo sobre la publicación de datos de pacientes.

Derecho a la privacidad y consentimiento informado. Los autores han obtenido el consentimiento informado de los pacientes o sujetos referidos en el artículo. Este documento obra en poder del autor de correspondencia.

\section{Bibliografía}

1. Kim JS. Acute abdominal pain in children. Pediatr Gastroenterol., Hepatol Nutr. 2013;16:219-24. Available from: https://synapse.koreamed.org/ DOlx.php?id=10.5223/pghn.2013.16.4.219.

2. Yi DY, Chang EJ, Kim JY, Lee EH, Yang HR. Age, predisposing disease and ultrasonographic findings in determining clinical outcome of acute acalculous inflammatory gallbladder disease in children. J Korean Med Sci. 2016;31:1617-23. Available from: https://synapse.koreamed.org/ search.php? where=aview\&id=10.3346/jkms.2016.31.10.1617\&code=0063JKMS\&vmode=FULL

3. Jeanty C, Derderian SC, Courtier J, Shinjiro H. Clinical management of infantile cholelithiasis. J Pediatr Surg. 2015;50:1289-92. Available from: https://www.sciencedirect.com/science/article/pii/S0022346814007027

4. Shaikh AA, Charles A, Domigo S, Schaub G. Gallbladder volvulus: report of two original cases and review of the literature. Am Surg. 2005;71:87. Avalable from: https://www.ingentaconnect.com/content/sesc/ tas/2005/00000071/00000001/art00017

5. Kimura T, Yonekura T, Yamauchi K, Kosumi T, Sasaki T, Kamiyama M. Laparoscopic treatment of gallbladder volvulus: a pediatric case report and literature review. Jour Lap Adv Surg Tech. 2008;18:330-334. Available from: https://www.liebertpub.com/doi/abs/10.1089/lap.2007.0057

6. Gupta V, Singh V, Sewkani A, Purohit D, Varshney R, Varshney S. Torsion of gall bladder, a rare entity: a case report and review article. Cases Journal. 2009;2:193. Available from: https://casesjournal.biomedcentral.com/articles/10.1186/1757-1626-2-193

7. Kitagawa H, Nakada K, Enami T, Yamaguchi T, Kawaguchi F. Nakada M, et al. Two cases of torsion of the gallbladder diagnosed preoperatively. J Pediatr Surg. 1997;32:1567-69. Available from: https://www.jpedsurg. org/article/S0022-3468(97)90454-1/abstract 